\title{
PRIMEIRA DESCRIÇÃO DO Lutzomyia longipalpis EM 11 MUNICÍPIOS DO ESTADO DE SÃO PAULO, 2014
}

\author{
FIRST DESCRIPTION OF Lutzomyia longipalpis IN 11 \\ SÃO PAULO STATE TOWNS, 2014
}

\author{
E. M. N. DE PAULA ${ }^{1}$, J. H. B. TOSCANO ${ }^{2 *}$, N. C. MARQUES ${ }^{2}$, M. B. D. OLIVARI ${ }^{2}$, B. F. IZOLA², \\ A. P. R. GRISÓLIO², R. B. MEIRELLES-BARTOLI ${ }^{1}$, A. A. B. CARVALHO²
}

\begin{abstract}
RESUMO
Lutzomyia longipalpis, popularmente conhecido como mosquito-palha, é o principal vetor da leishmaniose visceral (LV), enfermidade parasitária transmitida pela picada do inseto e cujas infecções variam de assintomática e leves (maioria dos casos) a fatais. Os casos de LV no Brasil estão em franco crescimento nas últimas décadas, principalmente pela expansão dos ambientes urbanos, invadindo áreas de vegetação naturalmente habitada pelo vetor e reservatórios silvestres. Tendo em vista o papel indispensável dos flebotomíneos na disseminação da leishmaniose, as pesquisas entomológicas são fundamentais, para se conhecer a fundo a distribuição e biologia deste díptero. Esse estudo objetivou descrever os municípios do Estado de São Paulo (ESP) que, pela primeira vez, relataram, em 2014, a presença do L. longipalpis. Trata-se de um estudo descritivo utilizando-se dados de pesquisas entomológicas da Superintendência de Controle de Endemias (SUCEN) do Estado de São Paulo. Ao todo 11 cidades do ESP registraram, em seus limites, a presença do L. longipalpis neste ano, sendo elas: Cordeirópolis, Valinhos, Álvares Florence, Floreal, Gastão Vidigal, General Salgado, Mira Estrela, Monções, Turmalina, Emilianópolis e Rancharia. Destes municípios, nenhum possui casos de leishmaniose em cães, entretanto, General Salgado registrou um caso de leishmaniose em ser humano.. Segundo o Ministério da Saúde, todos esses municípios são classificados epidemiologicamente como "silencioso receptivo vulnerável". Conlui-se, então, que 11 cidades relataram, pela primeira vez, Lutzomyia longipalpis apenas no ano de 2014. Embora praticamente ausentes os casos de LV nestes locais, reforça a importância dos estudos em relação a este díptero, o que permite apontar áreas receptivas à realização do inquérito amostral canino, orientando ações de controle do vetor e, consequentemente, da enfermidade.
\end{abstract}

PALAVRAS-CHAVE: VETOR. LEISHMANIOSE VISCERAL. VIGILÂNCIA EPIDEMIOLÓGICA. SAÚDE PÚBLICA VETERINÁRIA.

AGRADECIMENTOS: Superintendência de Controle de Endemias (SUCEN) do Estado de São Paulo

ÁREA TEMÁTICA: Zoonoses

\footnotetext{
${ }^{1}$ Universidade Federal de Goiás (UFG), Regional Jataí, Unidade Jatobá, Laboratório de Sanidade Animal

${ }^{2}$ Faculdade de Ciências Agrárias e Veterinárias da Universidade Estadual Paulista (UNESP) - Câmpus de Jaboticabal

* joaoh.toscano@gmail.com
} 